\title{
Constructing Near-BPS Skyrmions with Constant Baryon Densities
}

\author{
Luc Marleau*t \\ Département de Physique, de Génie Physique et d'Optique, Université Laval, Québec, Canada \\ E-mail: lmarleau@phy.ulaval.ca
}

\begin{abstract}
We propose to describe nuclei as near-BPS solitons emerging from a generalization of the Skyrme model in the regime where the sixth-order term and the mass term dominate. Emphasis is placed on the choice of the mass term in order to give baryon and energy densities approximately constant at the core of the nuclei, as opposed to the usual shell-like configurations found in most extensions of the Skyrme Model. Adding contributions from the rotational energy, Coulomb energy and isospin symmetry breaking, we reproduce the mass of the most abundant isotopes to rather good accuracy.
\end{abstract}

The European Physical Society Conference on High Energy Physics 18-24 July, 2013

Stockholm, Sweden

\footnotetext{
* Speaker.

†Work supported by the NSERC (Canada).
} 
Introduction and Motivation: Despite the successes of the Standard Model, we still have no clear description of the low-energy limit of QCD and especially on how quarks and gluons form nucleons and nuclei. An alternative approach, proposed by Skyrme [1] years ago, consists in an effective meson field theory from which baryons emerge as topological solitons. The Skyrme Model provides a relatively good picture of the nucleons yet, it seems unable to reproduce some of the features of nuclei and more precisely, their small binding energy. This last observation suggests that Skyrme-like models that nearly saturate the Bogomol'nyi bound (refered as near-BPS Skyrme Models) may be more appropriate to describe nuclei since their mass is roughly proportional to the baryon number. We propose a near-BPS model containing terms up to order six in derivatives of the pion fields, but where the usual nonlinear $\sigma$ and Skyrme terms are assumed to be relatively small. A special choice of mass term leading to analytical BPS-type solutions ensures approximately constant baryon density configurations, as opposed to the shell-like configurations found in the Skyrme Model and most of its extensions. We proceed to compute the binding energy per nucleon $B / A$ and compare our results with experimental data.

The near-BPS Skyrme Model: We consider an extension of the original Skyrme Model which is the most general $S U(2)$ model with at most two time derivatives

$$
\mathscr{L}=-\mu^{2} V(U)-\alpha \operatorname{Tr}\left[L_{\mu} L^{\mu}\right]+\beta \operatorname{Tr}\left(f_{\mu v} f^{\mu v}\right)-(3 / 512) \lambda^{2} \operatorname{Tr}\left(f_{\mu v} f^{v \lambda} f_{\lambda}^{\mu}\right)
$$

where $f_{\mu v}=\left[L_{\mu}, L_{v}\right]$ with $L_{\mu}=U^{\dagger} \partial_{\mu} U$, the left-handed current of the meson fields represented by the $S U(2)$ matrices $U=\phi_{0}+i \tau_{i} \phi_{i}$ which obey the nonlinear condition $\phi_{0}^{2}+\phi_{i}^{2}=1$. The constants $\mu$, $\alpha, \beta$, and $\lambda$ are free parameters but we shall be interested in the regime where $\mu$ and $\lambda$ dominate as opposed to the Skyrme Model where $\mu=\lambda=0$. The first term, the so-called mass term, is often added to take into account chiral symmetry breaking so that it generates a pion mass term for small fluctuations of the chiral field in $V(U)$. The next two terms are the nonlinear $\sigma$ and the Skyrme terms respectively. Finally, the term of order six in derivatives of the pion fields is equivalent to $\mathscr{L}_{J 6}=-\frac{\varepsilon_{6}}{4} \mathscr{B}^{\mu} \mathscr{B}_{\mu}$ that was first proposed to take into account $\omega$-meson interactions. Here, $\mathscr{B}^{\mu}=1 /\left(24 \pi^{2}\right) \varepsilon^{\mu \nu \rho \sigma} \operatorname{Tr}\left(L_{v} L_{\rho} L_{\sigma}\right)$ is the topological (baryon) current density, whose time component serves to characterize solutions by a conserved topological charge identified to baryon number or mass number in the context of nuclei, $A=\int d^{3} r \mathscr{B}^{0}$.

The model (1) has been studied extensively but it is only recently that the regime $\alpha, \beta \sim 0$ became of interest when Adam et al. [2] proposed a simpler version of this model, setting $\alpha=$ $\beta=0$. The classical solutions turn out to be BPS solitons in this case but as such they have no binding energy. Also, without the nonlinear $\sigma$ and the Skyrme terms, the link with pion physics seems to be severed. So in order to get closer to saturation of Bogomol'nyi bound and restore this link, a more realistic approach was proposed in Refs. [3] where $\alpha$ and $\beta$ terms are assumed to be relatively small but non zero. Unfortunately, most models display shell-like baryon and energy densities whereas nuclear matter is known to be uniformly distributed inside a nucleus. Since the form of the potential is instrumental in the determination of the solution, we propose here a more appropriate mass term

$$
V_{\mathrm{BeM}}(U)=\frac{1792}{45} \operatorname{Tr}\left[U_{+} U_{-}^{3}\right] \frac{\left(1-(14 / 5) \ln \left(\operatorname{Tr}\left[U_{-}\right]\right)\right)}{1-\sqrt{1-(14 / 5) \ln \left(\operatorname{Tr}\left[U_{-}\right]\right)}}
$$


where $U_{ \pm}=\left(2 I \pm U \pm U^{\dagger}\right) / 8$. The goal here is to demonstrate that it is possible to construct an effective Lagrangian which leads to an approximately constant density configurations and still preserves and even improves the agreement with nuclear mass data.

The solutions take the general form $U=\cos F+i \hat{\mathbf{n}} \cdot \tau \sin F$ where $\hat{\mathbf{n}}$ is the unit vector $\hat{\mathbf{n}}=$ $(\sin \Theta \cos \Phi, \sin \Theta \sin \Phi, \cos \Theta)$ and $F, \Theta$, and $\Phi$ depend, in general, on the position variables $r, \theta$, and $\phi$. Since we are interested in the limit where $\alpha$ and $\beta$ are small, we first consider a most simple solution for $\alpha=\beta=0$ that have axial symmetry, i.e. $F=F(r), \Theta=\theta$, and, $\Phi=n \phi$ where $n$ corresponds to the baryon number $A$. Minimizing the static energy leads to the differential equation for $F$ whose solution take a simple analytical form $F_{\mathrm{BeM}}(x)=2 \arcsin \left[\exp \left(-x^{2}-7 x^{4} / 5\right)\right]$ where $x=(\mu / 18 n \lambda)^{1 / 3} r$. In order to estimate the nuclear masses, we then switch on the nonlinear $\sigma$ and Skyrme terms and compute in addition to the static energy $E_{\mathrm{S}}:(1)$ the (iso)rotational energy $E_{\mathrm{r}}$ through the quantization of the (iso)rotational modes, (2) the Coulomb energy $E_{\mathrm{C}}$ caused by the charge distribution inside the nucleus and (3) an isospin breaking term $E_{\mathrm{I}}$ to account for protonneutron mass difference. The mass of a nucleus, $E_{\mathrm{tot}}=E_{\mathrm{s}}+E_{\mathrm{r}}+E_{\mathrm{C}}+E_{\mathrm{I}}$, then depends on the four parameters of the model and the baryon number (see Ref. [4] for more details).

Results and discussion: At this point the values of the parameters $\mu, \alpha, \beta$, and, $\lambda$ remain to be fixed. Using the data from the 140 most stable isotopes, we fit the parameters with our expression for the mass. We get $\mu=1.3264 \times 10^{4} \mathrm{MeV}^{2}, \lambda=5.7037 \times 10^{-3} \mathrm{MeV}^{-1}$ and verify that $\alpha$ and $\beta$ turn out to be quite small with values $\alpha=1.48244 \times 10^{-3} \mathrm{MeV}^{2}$ and. $\beta=1.20427 \times 10^{-8}$. This set of parameters results in values for the masses $E_{\text {tot }}$ that are accurate to at least $0.8 \%$, even for heavier nuclei, and within $10 \%$ for binding energy per nucleon $B / A$. The predictions are indeed surprisingly good compared to that of the Skyrme Model which overestimates the $B / A$ by at least an order of magnitude. Despite this success, further analysis are required to account for: (a) the size of nuclei which is proportional to $A^{\frac{1}{3}}$ but is slightly too large $r_{0}=1.91 \mathrm{fm}$ as compared to the experimental value $1.23 \mathrm{fm}$, (b) the rotational energy is small especially for large nucleus, (c) the pion decay constant would be $F_{\pi}=4 \sqrt{\alpha} \ll 186 \mathrm{MeV}$ and $m_{\pi}=\mu \sqrt{2 / \alpha} \gg 138 \mathrm{MeV}$ so that the link to soft-pion physics is yet unclear and (d) several aspects of the model (e.g. magnetic moments, vibrational and rotational excitations....) remain to be studied. Nonetheless, we have demonstrated that it is possible to construct constant core baryon and charge densities for all $A$. Although it remains a prototype model, it provides a surprising accurate description of $B / A$ and other properties of the nuclei. More generally, it clearly supports the idea that nuclei could be near-BPS Skyrmions.

\section{References}

[1] T.H.R. Skyrme, Proc. R. Soc. A 260, 127 (1961)

[2] C. Adam, J. Sanchez-Guillen and A. Wereszczynski, Phys.Lett.B691:105-110, 2010.

[3] E. Bonenfant, L. Marleau, Phys.Rev. D 82, 054023 (2010) ; E. Bonenfant, L. Harbour, L. Marleau ibid.85, 114045 (2012).

[4] M.O. Beaudoin, L. Marleau, arXiv:1305.4944. 